\title{
Anthocyanins From the Fruit of Vitis coignetiae Pulliat Potentiate the Cisplatin Activity by Inhibiting PI3K/Akt Signaling Pathways in Human Gastric Cancer Cells

\author{
Jing Nan $\mathrm{Lu}^{1 * * *}$, Won Sup Lee ${ }^{1 *}$, Arulkumar Nagappan ${ }^{1}$, Seong-Hwan Chang ${ }^{2}$, Yung Hyun $\mathrm{Choi}^{3}$, Hye Jung Kim ${ }^{4}$, \\ Gon Sup Kim ${ }^{5}$, Chung Ho Ryu ${ }^{6}$, Sung Chul Shin ${ }^{7}$, Jin-Myung Jung ${ }^{8}$, Soon Chan Hong ${ }^{9}$
}

${ }^{1}$ Department of Internal Medicine, Gyeongsang National University School of Medicine, Jinju, ${ }^{2}$ Department of Surgery, Konkuk University School of Medicine, Seoul, ${ }^{3}$ Department of Biochemistry, Dongeui University College of Oriental Medicine and Department of Biomaterial Control (BK21 program), Dongeui University Graduate School, Busan, ${ }^{4}$ Department of Pharmacology, Gyeongsang National University School of Medicine, ${ }^{5}$ School of Veterinary Medicine, ${ }^{6}$ Division of Applied Life Science (BK 21 Program), Institute of Agriculture and Life Science, ${ }^{7}$ Department of Chemistry, Research Institute of Life Science, Gyeongsang National University, Departments of ${ }^{8}$ Neurosurgery and ${ }^{9}$ Surgery, Institute of Health Sciences, Gyeongsang National University School of Medicine, Jinju, Korea

Background: Cisplatin (cis-diaminedichloroplatinum, CDDP) is a widely used chemotherapeutic agent for the treatment of many cancers. However, initial resistance to CDDP is a serious problem in treating these cancers. Vitis coignetiae Pulliat (Meoru in Korea) have shown anti-nuclear factor kappa B and anti-epidermal growth factor receptor activities in cancer cells.

Methods: In this study, in order to seeking an approach to increase the anti-cancer effects of CDDP with natural products. Here, we investigated anthocyanins isolated from Vitis coignetiae Pulliat (anthocyanidins isolated from meoru, AIMs) can enhance anti-cancer effects of cisplatin (CDDP) in stomach cancer cells. The cell viability of SNU-1 and SNU-16 cells after treated with AIMs and CDDP were analyzed by MTT assay. The expressions of Akt and X-linked inhibitor of apoptosis protein (XIAP) proteins were examined by western blot in AIMs- and CDDP-treated cells.

Results: We found that AIMs enhanced anticancer effects of CDDP, which activity was additive but not synergistic. AlMs suppressed Akt activity of the cancer cells activated by CDDP. AIMs also suppressed in XIAP an anti-apoptotic protein.

Conclusions: This study suggests that the anthocyanins isolated from fruits of Vitis coignetiae Pulliat enhanced anti-cancer effects of CDDP by inhibiting Akt activity activated by CDDP.

(J Cancer Prev 2015;20:50-56)

Key Words: Anthocyanins, Cisplatin, Stomach neoplasms

\section{INTRODUCTION}

Throughout most of the twentieth century, gastric cancer has been one of the leading causes of cancer death worldwide. ${ }^{1,2}$ The main treatment is surgical resection, but most of advanced gastric cancer patients require systemic chemotherapy after surgery for adjuvant or palliative purposes. As systemic chemotherapy, cisplatin (cis-diaminedichloroplatinum, CDDP) is a widely used. In general, CDDP binds to DNA efficiently to form a variety of monoadducts and cross links either between adjacent bases on the same strand or opposing strands of DNA. ${ }^{3,4}$ These DNA lesions contribute to the cytotoxicity of CDDP through blocking DNA replication and stimulating signals for apoptosis. ${ }^{5}$ However, the resistance to CDDP is developing while on chemotherapy,

Received February 2, 2015, Revised February 27, 2015, Accepted February 27, 2015

Correspondence to: Won Sup Lee

Department of Internal Medicine, Institute of Health Sciences, Gyeongsang National University School of Medicine, 79 Gangnam-ro, Jinju 660-702, Korea

Tel: +82-55-750-8733, Fax: +82-55-758-9122, E-mail: lwshmo@gnu.ac.kr

*These two authors contributed equally to this work.

**Current affiliation: The Fifth Hospital of Shijiazhuang, Shijiazhuang, 050021, China

Copyright (C) 2015 Korean Society of Cancer Prevention

(c) This is an Open Access article distributed under the terms of the Creative Commons Attribution Non-Commercial License (http://creativecommons. org/icenses/by-nc/3.0) which permits unrestricted non-commercial use, distribution, and reproduction in any medium, provided the original work is properly cited. 
which is the major reason for the failure in chemotherapy. CDDP resistance can be the final phenomenon from multifactorial molecular mechanisms, which is not fully elucidated. One mechanism of resistance may be mediated through enhanced anti-apoptotic activity by activating Akt activity. ${ }^{6}$ In practice, to overcome initial resistance to CDDP, we usually add another cytotoxic chemotherapy that has different mechanisms of action and resistance.

As advances in medical science, our lifespan has been extended. The population of elderly cancer patients and their cancer-related mortality are expected to increase because the elderly have a high risk for cancer development. ${ }^{1}$ However, these elderly patients cannot tolerate the conventional multi-combination chemotherapy. In turn, the most of elderly patients have experienced serious side effects from this combination chemotherapy. Some of these patients die of complications of chemotherapy. In this context, we need to find out less toxic agents in controlling cancer and enhancing the anti-cancer effects of the single anti-cancer agent.

Recently dietary agents are known to safely modulate physiological function and enhance anti-cancer activity. ${ }^{7-9}$ Apoptosis, is one of the anti-cancer mechanism of phytochemicals from fruits and vegetables, and is an active-energy requiring process (a type I programmed cell death) with distinctive phenotype such as blebbing, cell shrinkage, nuclear fragmentation, chromatin condensation, and chromosomal DNA fragmentation. ${ }^{10,11}$ Its serve as defense mechanism for cancer development by eliminating damaged cells which are prone to develop cancer. Vitis coignetiae Pulliat (Meoru in Korea) traditionally has been used in Korean folk medicine for the treatment of inflammatory lesions and cancer. It contains abundance of anthocyanins belonging to a class of flavonoids. Recently, in vitro and in vivo anti-cancer activities of anthocyanins have been demonstrated regarding anti-angiognesis and cancer invasion. ${ }^{12,13}$ We previously suggested that the anthocyanins isolated from Vitis coignetiae Pulliat (anthocyanidins isolated from meoru, AIMs) may induce apoptosis by suppressing Akt activity. ${ }^{14}$ Here, we determine whether AIMs can enhance anti-cancer effect of CDDP and investigate its involved mechanisms.

\section{MATERIALS AND METHODS}

\section{Cell culture and chemicals}

Two of the gastric cancer cell lines, SNU-1, SNU-16 cells were obtained from the Laboratory of Cell Biology at the Cancer Research Institute in Seoul National University College of
Medicine. They were cultured in RPMI-1640 supplemented with $10 \%$ fetal bovine serum (Gibco BRL, Carlsbad, CA, USA), $100 \mathrm{U}$ of penicillin and $100 \mu \mathrm{g} / \mathrm{mL}$ of streptomycin at $37^{\circ} \mathrm{C}$ in the humidified atmosphere of $95 \%$ air and $5 \% \mathrm{CO}_{2}$ in an incubator. Molecular mass markers for proteins were obtained from Pharmacia Biotech (Saclay, France). Antibodies against phosphoAkt (Ser473), Akt 1/2/3, X-linked inhibitor of apoptosis protein (XIAP), and procaspase 3 were purchased from Santa Cruz Biotechnology Inc. (Santa Cruz, CA, USA). Antibodies against phospho-Akt (Thr 308), and phospho-p53 (Ser 15) were purchased from Cell Signaling Technology, Inc. (Beverly, MA, USA). Antibody against poly (adenosine diphosphate-ribose) polymerase (PARP) was purchased from BD Biosciences Pharmingen (San Diego, CA, USA). Antibody against $\beta$-actin was from Sigma (Beverly, MA, USA). Peroxidase-labeled donkey anti-rabbit and sheep antimouse immunoglobulin, and an enhanced chemiluminescence (ECL) kit were purchased from Amersham (Arlington Heights, IL, USA). All other chemicals not specifically cited here were purchased from Sigma Chemical Co. (St. Louis, MO, USA).

\section{Preparation of anthocyanins}

Fruits of Vitis coignetiae Pulliat were collected at Jiri Mountain, Republic of Korea. Anthocyanins were isolated and characterized as previously described. ${ }^{15}$ The composition of AIMs was as follows: delphinidin-3, 5-diglucoside : cyanidin-3, 5-diglucoside : petunidin-3, 5-diglucoside : delphinidin-3-glucoside : malvdin-3, 5-diglucoside : peonidin-3, 5-diglucoside : cyanidin-3-glucoside : petunidin-3-glucoside : peonidin-3-glucoside : malvidin-3-glucoside $=3.5: 3.4: 7.1: 23.9: 8.0: 9.6: 9.1: 16.1: 5.7: 13.4$.

\section{Cell proliferation assays}

For the cell viability assay, the cells were seeded onto 24-well plates at a concentration of $5 \times 10^{4}$ cells $/ \mathrm{mL}$, grown to $70 \%$ confluence and then treated with the indicated concentration of AIMs for 48 hours. Control cells were supplemented with media containing $0.1 \%$ dimethyl sulfoxide (vehicle control). Following treatment, cell viability was determined by MTT assays.

\section{Western blotting}

The procedure was performed as described previously. ${ }^{16}$ In brief, the concentrations of cell lysate proteins were determined by means of the Bradford protein assay (Biorad Lab, Ricmond, CA, USA) using bovine serum albumin as the standard. The protein $(30 \mu \mathrm{g})$ was resolved by electrophoresis, electrotransferred to a polyvinylidene difluoride membrane (Millipore, Bedford, MA, USA), and then incubated with primary antibodies followed by 
secondary antibody conjugated to peroxidase. Blots were developed with an ECL detection system.

\section{Statistics}

Each experiment was performed in triplicate. The results were
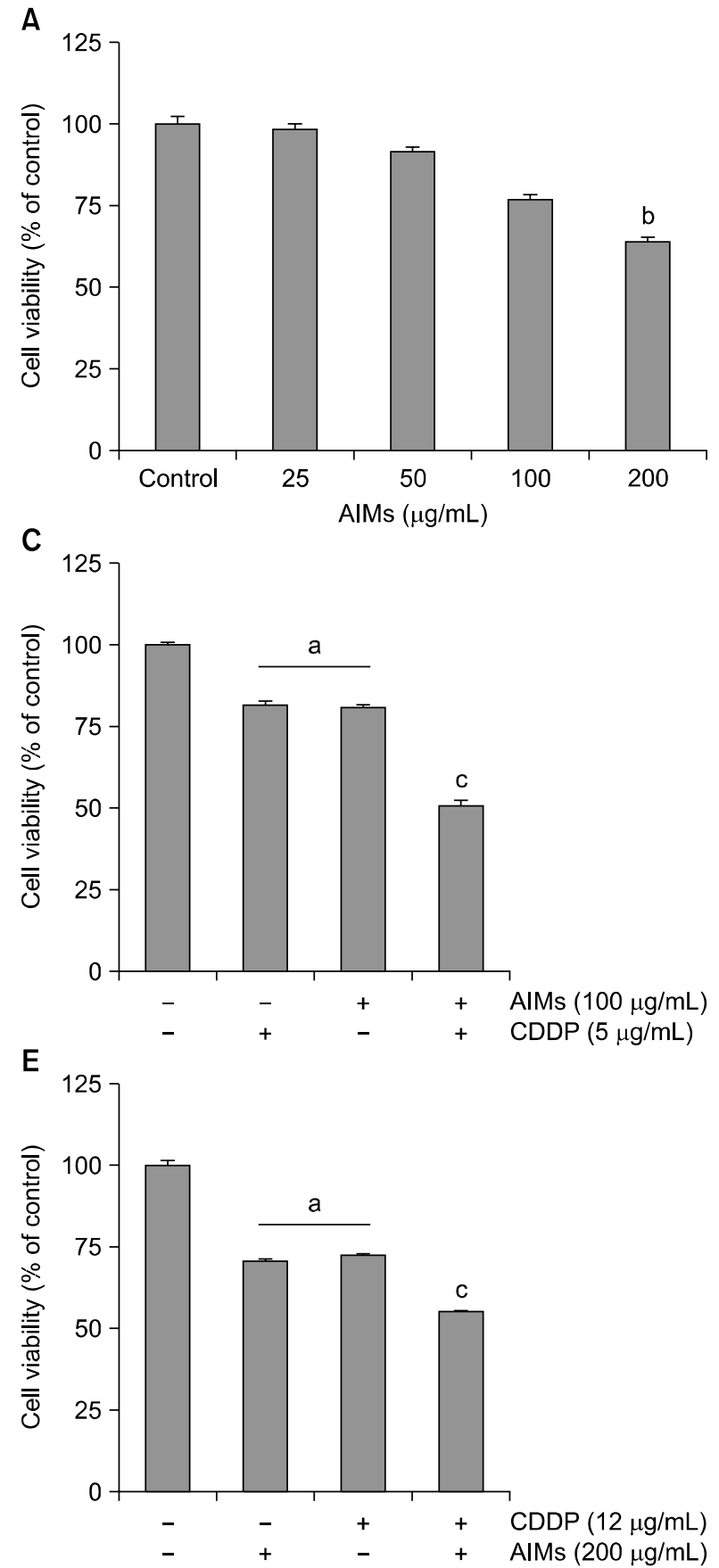

expressed as means \pm SD. Significant differences were determined using the one-way analysis of variance with post-test Newman-Keuls for the cases at least three treatment groups and Student's $t$-test for two group comparison. Statistical significance was defined as $P<0.05$. Statistical analysis was performed using
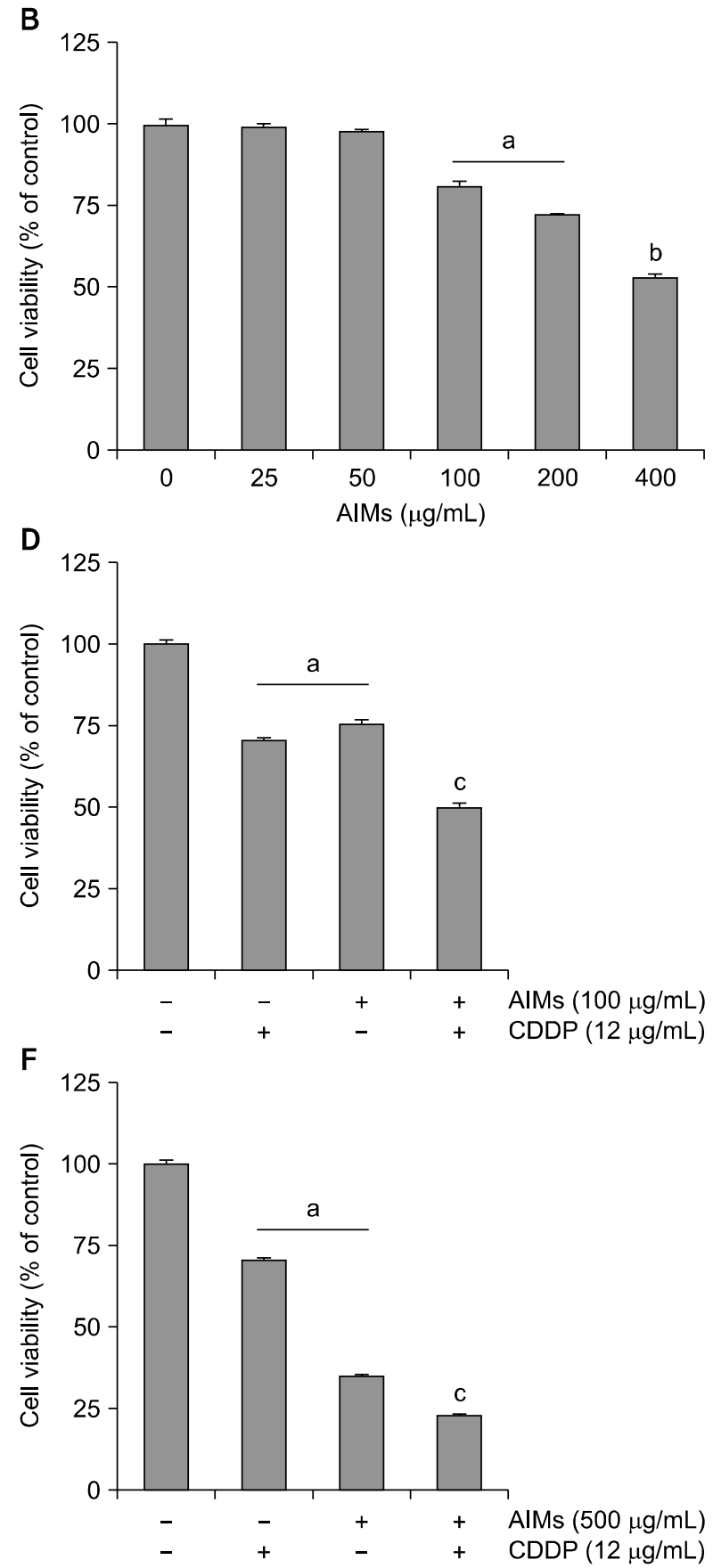

Figure 1. The inhibitory effects of anthocyanins isolated from Vitis coignetiae Pulliat (anthocyanidins isolated from meoru, AIMs) and/or cis-diaminedichloroplatinum (CDDP) on SNU-1, and SNU-16 human gastric cancer cells. Cell proliferation was assessed by MTT assay. (A, B) SNU-1 cells were treated with indicated concentrations of AIMs for 24 hours. (C-F) SNU-16 cells were treated with indicated concentrations of AIMs for 24 hours. The data are shown as means \pm SD of three independent experiments. ${ }^{\mathrm{a}} P<0.05$ versus the untreated group. ${ }^{\mathrm{b}} P<$ 0.01 versus the untreated group. ${ }^{c} P<0.05$ versus the CDDP-treated group. 
A

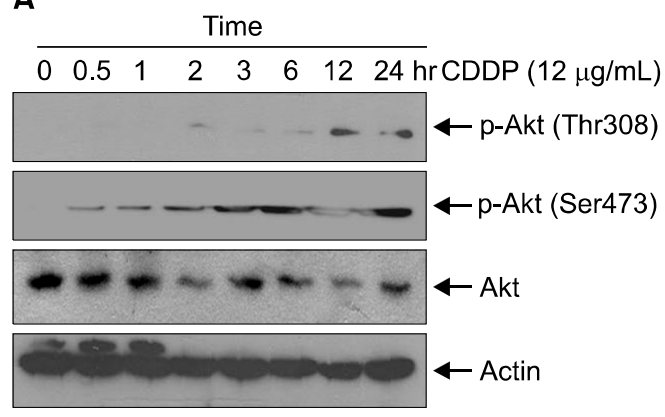

B

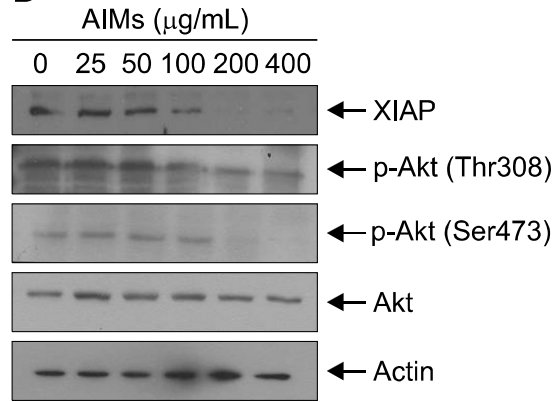

Figure 2. The effects of cis-diaminedichloroplatinum (CDDP) and/or anthocyanins isolated from Vitis coignetiae Pulliat (anthocyanidins isolated from meoru, AIMs) on Akt activity in SNU-16 cells. The expressions of phosphorylated Akt were assessed by Western blot analysis. (A) The cells were treated with CDDP $(12 \mu \mathrm{g} / \mathrm{mL})$ at indicated times. (B) Tumor necrosis factor $(10 \mathrm{ng} / \mathrm{mL})$ and CDDP were pretreated for 1 hour before AIMs treatment. Equal amounts of the cell lysate were separated by SDS-polyacrylamide gels and then transferred to nitrocellulose membranes and probed with the indicated antibodies and detected by an enhanced chemiluminescence detection system. Each result is a representative of at least two independent experiments. XIAP, X-linked inhibitor of apoptosis protein.

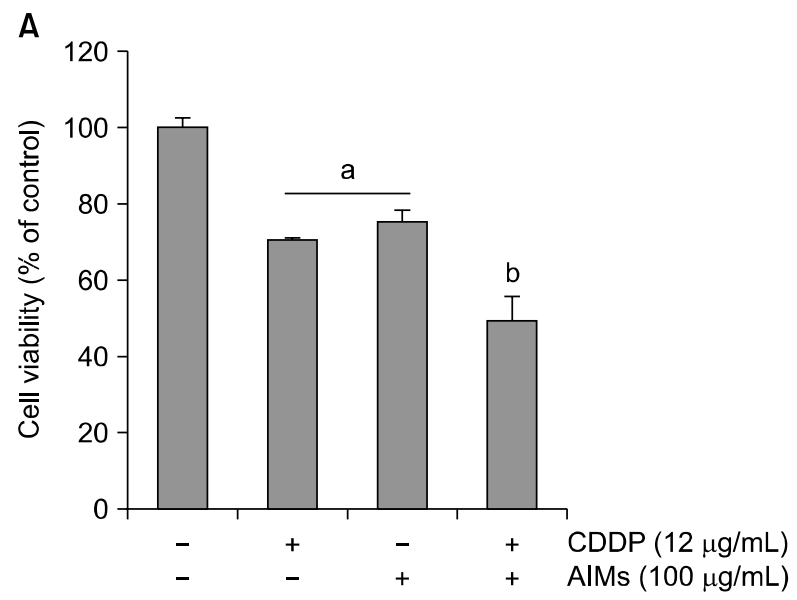

B

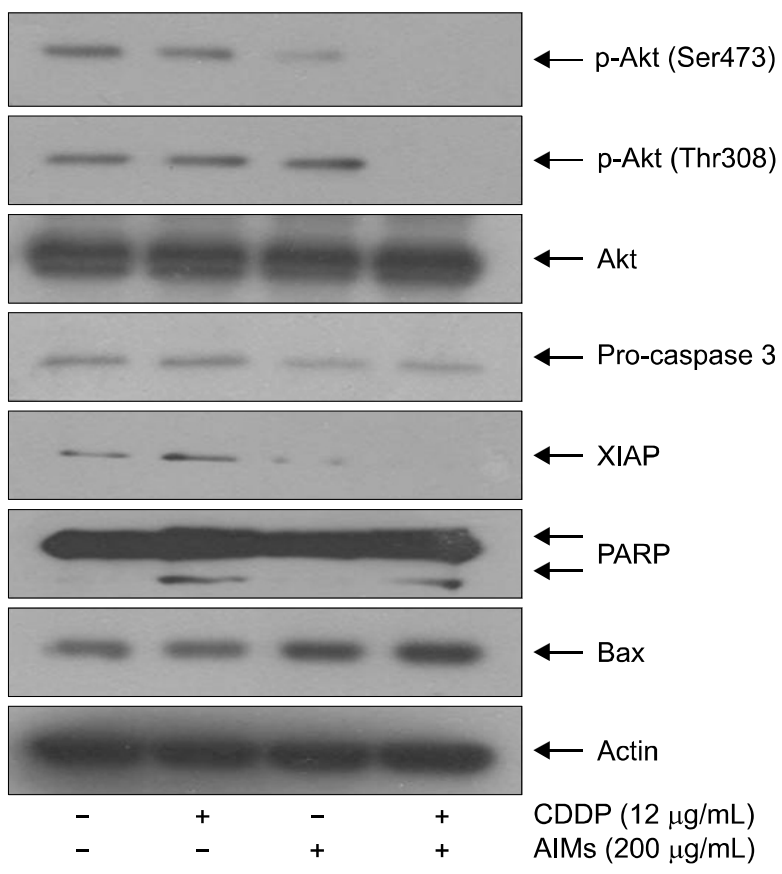

Figure 3. Effects of anthocyanins isolated from Vitis coignetiae Pulliat (anthocyanidins isolated from meoru, AIMs) on cis-diaminedichloroplatinum (CDDP)-induced apoptosis by regulating p-Akt in SNU-16 cells. SNU-16 cells were seeded at the density of $5 \times 10^{4}$ cells $/ \mathrm{mL}$. The cells were treated with CDDP $(12 \mu \mathrm{g} / \mathrm{mL})$ and/or AIMs $(200 \mu \mathrm{M})$ for 24 hours. (A) Cell proliferation was assessed by MTT assay. The data are shown as mean $\pm \mathrm{SD}$ of three independent experiments. ${ }^{\mathrm{a}} P<0.05$ versus the untreated group. ${ }^{\mathrm{b}} P<0.05$ versus the CDDP-treated group. (B) Equal amounts of cell lysate $(30 \mu \mathrm{g})$ were resolved by SDS polyacrylamide gels and transferred onto nitrocellulose membranes. The membranes were probed with the indicated antibodies and detected by the enhanced chemiluminescence detection system. The results are from a representative experiment of at least three independent experiments that showed similar patterns. XIAP, X-linked inhibitor of apoptosis protein; PARP, poly adenosine diphosphate-ribose polymerase. 
IBM SPSS version 21.0 software (IMB Co., Armonk, NY, USA).

\section{RESULTS}

1. Anthocyanins isolated from Vitis coignetiae Pulliat (AIMs) suppress the proliferation of SNU-1 and SNU-16 cells and enhanced the anticancer effects cis-diaminedichloroplatinum

First, we assessed the effects of AIMs on the cell growth. MTT assay revealed that AIMs suppressed the proliferation of both the SNU-1 and SNU-16 cells in a dose-dependent manner (Fig. 1A and 1B). The degrees to inhibitory effects on the cell proliferation are similar in both cell lines. We previously found that SNU-1 cells are more sensitive to CDDP than SNU-16 cells at the high concentration that can induce apoptosis. At $100 \mu \mathrm{g} / \mathrm{mL}$ of AIMs, the difference in sensitivity to AIMs between SNU-1 and SNU-16 cells was not so much (Fig. 1C and 1D). Next, we checked the effects of CDDP and AIMs in the higher dose. As we previously showed higher CDDP $(12 \mu \mathrm{g} / \mathrm{mL})$ did not increased cell death in SNU-16 cells, but the increase in dose to $500 \mu \mathrm{g} / \mathrm{mL}$ of AIMs enhanced anticancer effects on SNU-16 cells, and also enhanced cytotoxicity of CDDP, but the enhanced cytotoxicity did not meet synergism (Fig. 1E and 1F) These results suggest that AIMs have anti-cancer cancer effects on SNU-16 cells which is resistant to CDDP.

2. cis-Diaminedichloroplatinum increased Akt activity, which was suppressed by anthocyanins isolated from Vitis coignetiae Pulliat

In this study, we first tested whether CDDP increases Akt activities. As shown in Figure 2A CDDP begin to increase Akt activities 2 hours after CDDP treatment. Next, we first tested whether AIMs inhibits the increased Akt activities. Western blot analysis revealed that AIMs inhibited the increased Akt activities in a dose-dependent manner (Fig. 2B). This study indicated that the inhibitory effects of AIMs on Akt may influence the anticancer effects of CDDP.

3. Anthocyanins isolated from Vitis coignetiae Pulliat enhanced cis-diaminedichloroplatinuminduced apoptosis by suppression of phosphorylation of Akt

Previously we reported that AIMs can induce apoptosis by reducing Bcl-2, XIAP through the inhibition of Akt-phosphorylation. ${ }^{14}$ To determine that AIMs enhance CDDP-induced apoptosis, we performed cell viability assay. As shown in Figure 3A, AIMs enhanced cytotoxicity of CDDP. To confirm this finding at the molecular level, we performed western blot analysis for apoptosis related factors and p-Akt. The suppression of Akt phosphorylation led to inhibition of XIAP and activation of apoptosis related enzyme (PARP and caspase 3). We found that AIMs enhance the cytotoxicity of CDDP by suppressing XIAP and Akt. This result suggests that AIMs can enhance the sensitivity of CDDP in p-53 mutant SNU-16 cells which is resistant to CDDP.

\section{DISCUSSION}

Recently, phytochemicals or food substances have been known to safely modulate and enhance anti-cancer activity. ${ }^{8,17,18}$ We previously reported that AIMs can enhance apoptosis by suppression of Akt and anti-apoptotic proteins such as Bcl-2, and XIAP. In addition, Akt activation is accepted as one of major determinants of CDDP resistance in cancer cells. Moreover, Akt plays a critical role in regulating cell proliferation and apoptosis. Evidence suggest that Akt is also an upstream signal controlling Bcl-2 and IAP family members such as pro-apoptotic Bad and anti-apoptotic XIAP proteins, and its activity was regulated by phosphorylation. ${ }^{19}$ Therefore, it is reasonable to investigate whether AIMs can enhance the CDDP sensitivity in the SNU-16

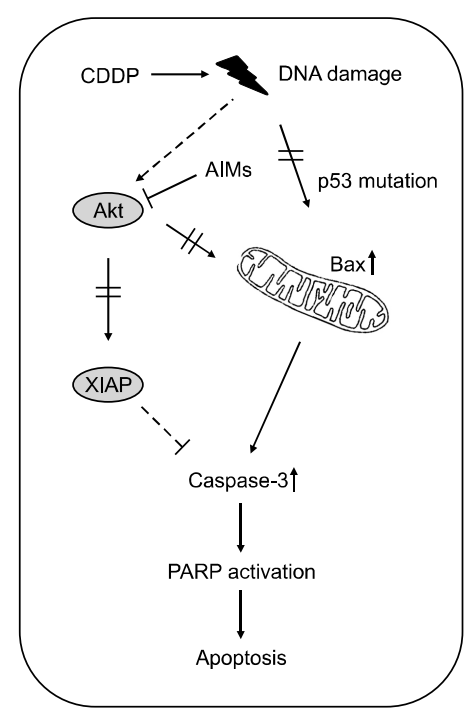

Figure 4. Schematic representation of anthocyanins isolated from Vitis coignetiae Pulliat (anthocyanidins isolated from meoru, AIMs) enhances the cis-diaminedichloroplatinum (CDDP)-induced apoptosis in SNU-16 human gastric carcinoma cells. AIMS enhanced the CDDP-induced apoptosis by induction of Bax as well as suppressing X-linked inhibitor of apoptosis protein (XIAP) through the inhibition of Akt in p53 mutant SNU-16 cells. Also, AIMS Taken together, this study suggests that AIMs enhance anti-cancer effects of CDDP in p53 mutant SNU-16 human gastric cancer cells. PARP, Poly adenosine diphosphate-ribose polymerase. 
which is resistant to CDDP. This study suggested that an increase in Akt activity is a cause of the failure of CCDP-induced apoptosis. Here, we need to check whether AIMs can suppress the increased Akt activity induced by CDDP. Consistent with previous studies showing that AIMs induce apoptosis by inhibiting Akt-phosphorylation, ${ }^{14}$ here AIMs suppressed the Akt-phosphorylation induced by CDDP. In addition to anti-Akt activity, AIMs has anti-epidermal growth factor receptor (EGFR) effects. ${ }^{20}$ The anti-EGFR effects also contribute to suppress XIAP, an antiapoptotic protein that we have shown in Figure 3B. We also tested another p-53 mutant MKN-28 human gastric cancer cells. The anthocyanins also enhance CDDP sensitivity of MKN-28 cells which is aggressive phenotypes and resistant to CDDP (data not shown). This is supporting our data that AIMs can enhance CDDP activity that is resistant to CDDP. In the present study, we have showed that AIMs enhances the CDDP-induced apoptosis in in p53 mutant SNU-16 cells (Fig. 4). In detail, AIMS enhanced the CDDP-induced apoptosis by induction of Bax as well as suppressing XIAP through the inhibition of Akt in SNU-16 cells. However, AIMs could not enhance sensitivity in p-53 wild SNU-1 cells. It is still under evaluation why the AIMs did not enhance anti-cancer effects of CDDP in SNU-1 cells.

The limitation is that there may be many other ways to enhance CDDP sensitivity using phytochemicals. Here, we are only focusing on Akt activities. Actually we previously found that p53 restoration can significantly enhance CDDP's cytotoxic activities. ${ }^{21}$ Therefore, further investigation is required to find another way to enhance CDDP activities. Another limitation is that the maximum concentration used in this study appears to be too high to perform in vivo study. Actually we have shown the anti-cancer effects in vivo with AIMs. ${ }^{22}$ In addition, the maximal dose of the anthocyanins used in this study is consistent with those in many other studies on the anti-tumor effect of anthocyanins in culture cells. ${ }^{23-25}$

In conclusion, our results suggest that AIMs can enhance cisplatin induced apoptosis by suppression of Akt and XIAP in CDDP-resistant SNU-16 human gastric cancer cells.

\section{ACKNOWLEDGMENTS}

This study was supported by a grant from the National R\&D Program for Cancer Control, Ministry for Health, Welfare \& Family Affairs, Republic of Korea (0820050).

\section{CONFLICTS OF INTEREST}

No potential conflicts of interest were disclosed.

\section{REFERENCES}

1. Jung KW, Won YJ, Kong HJ, Oh CM, Seo HG, Lee JS. Prediction of cancer incidence and mortality in Korea, 2013. Cancer Res Treat 2013;45:15-21.

2. Kavousi A, Bashiri Y, Mehrabi Y, Etemad K, Teymourpour A. Identifying high-risk clusters of gastric cancer incidence in iran, 2004 - 2009. Asian Pac J Cancer Prev 2014;15:10335-7.

3. Zwelling LA, Anderson T, Kohn KW. DNA-protein and DNA interstrand cross-linking by cis- and trans-platinum(II) diamminedichloride in L1210 mouse leukemia cells and relation to cytotoxicity. Cancer Res 1979:39:365-9.

4. Fichtinger-Schepman AM, van der Veer JL, den Hartog JH, Lohman PH, Reedijk J. Adducts of the antitumor drug cis-diamminedichloroplatinum(II) with DNA: formation, identification, and quantitation. Biochemistry 1985;24:707-13.

5. Bottone MG, Soldani C, Veneroni P, Avella D, Pisu M, Bernocchi G. Cell proliferation, apoptosis and mitochondrial damage in rat B50 neuronal cells after cisplatin treatment. Cell Prolif 2008;41:506-20.

6. Li J, Feng Q, Kim JM, Schneiderman D, Liston P, Li M, et al. Human ovarian cancer and cisplatin resistance: possible role of inhibitor of apoptosis proteins. Endocrinology 2001;142:370-80.

7. Liu BL, Zhang X, Zhang W, Zhen HN. New enlightenment of French Paradox: resveratrol's potential for cancer chemoprevention and anti-cancer therapy. Cancer Biol Ther 2007;6:1833-6.

8. Hatcher H, Planalp R, Cho J, Torti FM, Torti SV. Curcumin: from ancient medicine to current clinical trials. Cell Mol Life Sci 2008:65:1631-52.

9. Salvioli S, Sikora E, Cooper EL, Franceschi C. Curcumin in cell death processes: a challenge for CAM of age-related pathologies. Evid Based Complement Alternat Med 2007;4:181-90.

10. Buendia B, Santa-Maria A, Courvalin JC. Caspase-dependent proteolysis of integral and peripheral proteins of nuclear membranes and nuclear pore complex proteins during apoptosis. J Cell Sci 1999;112:1743-53.

11. Kerr JF, Wyllie AH, Currie AR. Apoptosis: a basic biological phenomenon with wide-ranging implications in tissue kinetics. $\mathrm{Br} \mathrm{J}$ Cancer 1972;26:239-57.

12. Syed DN, Afaq F, Sarfaraz S, Khan N, Kedlaya R, Setaluri V, et al. Delphinidin inhibits cell proliferation and invasion via modulation of Met receptor phosphorylation. Toxicol Appl Pharmacol 2008;231:52-60.

13. Favot L, Martin S, Keravis T, Andriantsitohaina R, Lugnier C. Involvement of cyclin-dependent pathway in the inhibitory effect of delphinidin on angiogenesis. Cardiovasc Res 2003:59: 479-87.

14. Shin DY, Lee WS, Lu JN, Kang MH, Ryu CH, Kim GY, et al. Induction of apoptosis in human colon cancer HCT-116 cells by anthocyanins through suppression of Akt and activation of p38-MAPK. Int J Oncol 2009;35:1499-504.

15. Yun JW, Lee WS, Kim MJ, Lu JN, Kang MH, Kim HG, et al. Characterization of a profile of the anthocyanins isolated from 
Vitis coignetiae Pulliat and their anti-invasive activity on HT-29 human colon cancer cells. Food Chem Toxicol 2010;48:903-9.

16. Kim HJ, Tsoy I, Park JM, Chung JI, Shin SC, Chang KC. Anthocyanins from soybean seed coat inhibit the expression of TNF-alpha-induced genes associated with ischemia/reperfusion in endothelial cell by NF-kappaB-dependent pathway and reduce rat myocardial damages incurred by ischemia and reperfusion in vivo. FEBS Lett 2006;580:1391-7.

17. Lee HY, Oh SH, Woo JK, Kim WY, Van Pelt CS, Price RE, et al. Chemopreventive effects of deguelin, a novel Akt inhibitor, on tobacco-induced lung tumorigenesis. J Natl Cancer Inst 2005:97: 1695-9.

18. Kundu JK, Chun KS. The promise of dried fruits in cancer chemoprevention. Asian Pac J Cancer Prev 2014;15:3343-52.

19. Franke TF, Hornik CP, Segev L, Shostak GA, Sugimoto C. PI3K/Akt and apoptosis: size matters. Oncogene 2003;22:8983-98.

20. Lu JN, Lee WS, Kim MJ, Yun JW, Jung JH, Yi SM, et al. The inhibitory effect of anthocyanins on Akt on invasion and epithelial-mesenchymal transition is not associated with the an-
ti-EGFR effect of the anthocyanins. Int J Oncol 2014:44:1756-66.

21. Kim CW, Lu JN, Go SI, Jung JH, Yi SM, Jeong JH, et al. p53 restoration can overcome cisplatin resistance through inhibition of Akt as well as induction of Bax. Int J Oncol 2013;43:1495-502.

22. Lee YK, Lee WS, Kim GS, Park OJ. Anthocyanins are novel AMPK $\alpha 1$ stimulators that suppress tumor growth by inhibiting mTOR phosphorylation. Oncol Rep 2010;24:1471-7.

23. Boivin D, Blanchette M, Barrette S, Moghrabi A, Béliveau R. Inhibition of cancer cell proliferation and suppression of TNF-induced activation of NFkappaB by edible berry juice. Anticancer Res 2007;27:937-48.

24. Yeh CT, Yen GC. Induction of apoptosis by the Anthocyanidins through regulation of $\mathrm{Bcl}-2$ gene and activation of c-Jun N-terminal kinase cascade in hepatoma cells. J Agric Food Chem 2005;53: 1740-9.

25. Koide $T$, Kamei $H$, Hashimoto $Y$, Kojima $T$, Hasegawa $M$. Antitumor effect of hydrolyzed anthocyanin from grape rinds and red rice. Cancer Biother Radiopharm 1996;11:273-7. 